\title{
Effect of gum arabic, $\beta$-cyclodextrin, and sodium caseinate as encapsulating agent on the oxidative stability and bioactive compounds of spray-dried kenaf seed oil
}

\begin{abstract}
Kenaf seed oil is prone to undergo oxidation due to its high content of unsaturated fatty acids, thus microencapsulation stands as an alternative to protect kenaf seed oil from the adverse environment. This study primarily aimed to evaluate the oxidative stability of microencapsulated refined kenaf seed oil (MRKSO) by the use of gum arabic, $\beta$-cyclodextrin, and sodium caseinate as the wall materials by spray drying. Bulk refined kenaf seed oil (BRKSO) and MRKSO were kept at $65{ }^{\circ} \mathrm{C}$ for 24 days to evaluate its oxidative stability, changes of tocopherol and tocotrienol contents, phytosterol content, and fatty acid profile. The results showed that the peroxide value, $p$-Anisidine value, and total oxidation value of BRKSO were significantly higher than the MRKSO at day 24. The total tocopherol and tocotrienol contents were reduced $66.1 \%$ and $56.8 \%$ in BRKSO and MRKSO, respectively, upon the storage. There was a reduction of $71.7 \%$ and $23.5 \%$ of phytosterol content in BRKSO and MRKSO, respectively, upon the storage. The degradation rate of polyunsaturated fatty acids in BRKSO was higher than that of MRKSO. This study showed that the current microencapsulation technique is a feasible way to retard the oxidation of kenaf seed oil.
\end{abstract}

Keyword: Superconductivity; Tl-1212 Superconductor; Transport Critical Current Density; Graphene 